\title{
GEOMETRIC GENERA FOR AMPLE VECTOR BUNDLES WITH REGULAR SECTIONS
}

\author{
Antonio LANTERI*
}

\begin{abstract}
Let $X$ be a smooth complex projective variety of dimension $n \geq 3$. A notion of geometric genus $p_{g}(X, \mathcal{E})$ for ample vector bundles $\mathcal{E}$ of rank $r<n$ on $X$ admitting some regular sections is introduced. The following inequality holds: $p_{g}(X, \mathcal{E}) \geq h^{n-r, 0}(X)$. The question of characterizing equality is discussed and the answer is given for $\mathcal{E}$ decomposable of corank 2. Some conjectures suggested by the result are formulated.
\end{abstract}

\section{Introduction}

Recently the notion of curve genus for an ample vector bundle on a projective manifold attracted the attention of several authors ([LMS], [M2], [I] [FkI]). In particular, Ishihara [I] introduced the $c_{r}$-sectional genus, which generalizes at the same time several genera previously studied.

Let $X$ be a smooth complex projective manifold of dimension $n$ and let $\mathcal{E}$ be an ample vector bundle of rank $r \leq n-1$ on $X$ having a regular section, i. e., there exists a global section $s \in \Gamma(\mathcal{E})$ whose zero locus $Z=(s)_{0}$ is a smooth submanifold of $X$ of the expected dimension $n-r$. For example this condition is certainly satisfied if $\mathcal{E}$ is ample and spanned. In the setting of pairs $(X, \mathcal{E})$ as above another notion of genus (also generalizing the curve genus) can be introduced: the geometric

*The author is a member of G.N.S.A.G.A. of the Italian I.N. d. A.M. This paper was prepared in the framework of the National Research Project "Geometria algebrica, algebra commutativa e aspetti computazionali", supported by the M.U.R.S.T. of the Italian Government.

1991 Mathematics Subject Classification: 14F05, 14C20; secondary $14 \mathrm{~J} 40$. Servicio de Publicaciones. Universidad Complutense. Madrid, 2000 
genus $p_{g}(X, \mathcal{E})$, which is defined as $p_{g}(Z)=h^{n-r, 0}(Z)$ (see Section 1). Note that $Z$ represents $c_{r}(\mathcal{E})$ and that the normal bundle of $Z$ in $X$ is isomorphic to $\mathcal{E}_{Z}$. Hence, if $r=n-1$ adjunction gives

$$
2 p_{g}(X, \mathcal{E})-2=\left(K_{X}+c_{1}(\mathcal{E})\right) c_{n-1}(\mathcal{E})
$$

showing that $p_{g}(X, \mathcal{E})$ is simply the curve genus of $(X, \mathcal{E})$, according to the terminology of [LMS]. In particular, for $r=n-1$ the character $p_{g}(X, \mathcal{E})$ can be defined and analyzed without any assumption on the sections of $\mathcal{E}$; for interesting results in this direction see [M2]. On the contrary, I would like to stress that the existence of a regular section seems to be crucial for defining $p_{g}(X, \mathcal{E})$ when $r \leq n-2$.

Now, having defined $p_{g}(X, \mathcal{E})$ for any $r \leq n-1$, it follows from the Lefschetz-Sommese theorem that

$$
p_{g}(X, \mathcal{E}) \geq h^{n-r, 0}(X)
$$

and it is natural to ask when $(*)$ is an equality (see [LMS] for the case $r=n-1)$. The same question for the similar inequality involving the $c_{r}$-sectional genus of an ample and spanned vector bundle of rank $r$ on $X$ and the irregularity $h^{0,1}(X)$ is answered in [I, Theorem 2.2]. Here the situation looks much more difficult and rich.

The Koszul complex associated with $\mathcal{E}$ and $Z$ easily suggests a cohomological adjunction theoretic condition for equality holding in $(*)$ (Proposition (1.1)). In general this condition is only sufficient, but for line bundles having enough sections it is also necessary (Proposition (2.1)). Relying on this condition, all decomposable ample and spanned vector bundles $\mathcal{E}$ of rank $n-2$ on a projetive $n$-fold $X$ for which equality holds in (*) are classified (Theorems (3.2) and (3.3)). This is done by combining techniques and results on ample divisors with Sommese's result on the nonemptiness of suitable adjoint linear systems on a smooth projective threefold. Finally, some conjectures arising from this classification, in comparison with other results in the literature, are formulated (Section 4).

I am very grateful to Enrique Arrondo for his helpful comments on a preliminary version of this paper. 


\section{The geometric genus and its lower bound}

We work over the complex number field $\mathbb{C}$ and we use the standard notation from algebraic geometry. Let $X$ be a projective manifold of dimension $n$. If $\mathcal{E}$ is a vector bundle of $\operatorname{rank} r<n$ on $X$ the integer $n-r$ is called the corank of $\mathcal{E}$. The pull-back $i^{*} \mathcal{E}$ of the vector bundle $\mathcal{E}$ on $X$ by an embedding $i: Y \hookrightarrow X$ is usually denoted by $\mathcal{E}_{Y}$. We say that the vector bundle $\mathcal{E}$ is spanned to mean that it is generated by global sections. The canonical bundle of a smooth variety $X$ is denoted by $K_{X}$. Following current abuses, I do not distinguish between a line bundle and the corresponding invertible sheaf and I freely switch from the multiplicative to the additive notation for the tensor products of line bundles.

Let $\mathcal{E}$ be a vector bundle of rank $r \leq n-1$ on $X$. Let $s \in \Gamma(\mathcal{E})$ be a global section of $\mathcal{E}$. Then $s$ defines a homomorphism $\mathcal{O}_{X} \rightarrow \mathcal{E}$ by sending 1 to $s$. Let $\eta: \mathcal{E}^{\vee} \rightarrow \mathcal{O}_{X}$ be its dual homomorphism. The closed subscheme $Z:=(s)_{0}$ of $X$ defined by the ideal $\mathcal{I}=\operatorname{Im}(\eta) \subset \mathcal{O}_{X}$ is called the zero locus of $s$. A global section $s$ of $\mathcal{E}$ is called regular if $Z$ is a smooth subvariety of codimension $r$. If $Z$ is the zero locus of a regular section of $\mathcal{E}$ we set $p_{g}(Z)=h^{n-r, 0}(Z)=h^{0}\left(K_{Z}\right)$.

Throughout all the paper we assume that

(1.0) $\mathcal{E}$ is an ample vector bundle of rank $r \leq n-1$ on $X$ admitting a regular section.

Recall that ample and spanned vector bundles satisfy the latter condition, due to the Bertini theorem.

Now note that $Z$ being smooth, connected and of the expected dimension are all open conditions. Hence, under the assumption (1.0), the regular sections of $\mathcal{E}$ constitute an open dense ( $\mathbb{C}^{*}$-invariant) subset of $\Gamma(\mathcal{E})$. Then, by $\left[\mathrm{H}\right.$, p. 289 , Example 12.9.3] we see that $p_{g}(Z)=$ $h^{n-r}\left(\mathcal{O}_{Z}\right)$ is constant on the open subset of $\mathbb{P}(\Gamma(\mathcal{E}))$ parametrizing the zero loci of regular sections. We can thus define the geometric genus $p_{g}(X, \mathcal{E})$ of the ample vector bundle $\mathcal{E}$ on $X$ as in (1.0) as follows:

$$
p_{g}(X, \mathcal{E}):=p_{g}(Z)
$$

$Z$ being the zero locus of any regular section of $\mathcal{E}$. Here is the first result concerning $p_{g}(X, \mathcal{E})$.

(1.1) Proposition. Let $X$ be a smooth complex projective $n$-fold and 
let $\mathcal{E}$ be an ample vector bundle on $X$ as in (1.0). Then

$$
p_{g}(X, \mathcal{E}) \geq h^{n-r, 0}(X) .
$$

Moreover equality holds if $h^{i}\left(K_{X} \otimes \wedge^{r-i} \mathcal{E}\right)=0$ for $i=0, \ldots, r-1$.

Proof. Let $Z$ be the zero locus of a regular section of $\mathcal{E}$ and consider the Koszul complex associated to $Z$

$$
0 \rightarrow \mathcal{O}_{X} \rightarrow \mathcal{E} \rightarrow \wedge^{2} \mathcal{E} \rightarrow \ldots \rightarrow \operatorname{det} \mathcal{E} \rightarrow \operatorname{det} \mathcal{E}_{Z} \rightarrow 0,
$$

which is exact since $Z$ is smooth of the expected dimension. Twisting it by $K_{X}$ and recalling that $\mathcal{E}_{Z}$ is the normal bundle of $Z$ in $X$, by adjunction we get the exact sequence

$$
0 \rightarrow K_{X} \rightarrow K_{X} \otimes \mathcal{E} \stackrel{\rho_{r}}{\rightarrow} K_{X} \otimes \wedge^{2} \mathcal{E} \stackrel{\rho_{r-1}}{\longrightarrow} \ldots \stackrel{\rho_{2}}{\rightarrow} K_{X}+\operatorname{det} \mathcal{E} \stackrel{\rho_{3}}{\rightarrow} K_{Z} \rightarrow 0 .
$$

Letting $\mathcal{F}_{i}=\operatorname{Ker}\left(\rho_{i}\right), i=1, \ldots, r-1$, we have the following short exact sequences:

$$
\begin{aligned}
0 & \rightarrow \mathcal{F}_{1} \rightarrow K_{X}+\operatorname{det} \mathcal{E} \stackrel{\rho_{1}}{\longrightarrow} K_{Z} \rightarrow 0, \\
0 & \rightarrow \mathcal{F}_{i} \rightarrow K_{X} \otimes \wedge^{n-i} \mathcal{E} \stackrel{\rho_{i}}{\longrightarrow} \mathcal{F}_{i-1} \rightarrow 0 \\
0 & \rightarrow K_{X} \rightarrow K_{X} \otimes \mathcal{E} \stackrel{\rho_{r}}{\longrightarrow} \mathcal{F}_{r-1} \rightarrow 0
\end{aligned}
$$

Now consider the exact cohomology sequences they induce. By the Le Potier vanishing theorem [SS, Theorem 5.71] we have

$$
H^{q}\left(K_{X} \otimes \wedge^{p} \mathcal{E}\right)=0 \text { for } q+p>r \text { and } 1 \leq p \leq r .
$$

Thus, for $(q, p)=(1, r),(1.1 .1)$ gives $h^{1}\left(K_{X}+\operatorname{det} \mathcal{E}\right)=0$, and so we get from the first cohomology sequence

$$
p_{g}(Z)=h^{0}\left(K_{Z}\right) \geq h^{1}\left(\mathcal{F}_{1}\right)
$$

moreover this is an equality if $h^{0}\left(K_{X}+\operatorname{det} \mathcal{E}\right)=0$, as the same exact sequence shows. Similarly, by using (1.1.1) for $(q, p)=(2, r-1), \ldots,(r-$ $1,2)$, we obtain

$$
h^{1}\left(\mathcal{F}_{1}\right) \geq h^{2}\left(\mathcal{F}_{2}\right) \geq \cdots \geq h^{r-1}\left(\mathcal{F}_{r-1}\right)
$$


and finally, the last exact cohomology sequence combined with (1.1.1) for $(q, p)=(r, 1)$ gives

$$
h^{r-1}\left(\mathcal{F}_{r-1}\right) \geq h^{r}\left(K_{X}\right) .
$$

Note that by Serre and Hodge duality $h^{r}\left(K_{X}\right)=h^{n-r}\left(\mathcal{O}_{X}\right)=h^{n-r, 0}(X)$. So, putting together all the above inequalities we get $p_{g}(Z) \geq h^{n-r, 0}(X)$. In view of the definition of $p_{g}(X, \mathcal{E})$, this gives $(*)$. Moreover we have equality in (*) if (1.1.2), (1.1.4) and all inequalities in (1.1.3) are equalities. Coming back to the exact cohomology sequences we used it is immediate to see that these equalities are in turn implied by

$$
H^{0}\left(K_{X} \otimes \wedge^{r} \mathcal{E}\right)=H^{1}\left(K_{X} \otimes \wedge^{r-1} \mathcal{E}\right)=\ldots=H^{r-1}\left(K_{X} \otimes \mathcal{E}\right)=0 .
$$

Let things be as in Proposition (1.1). By the Lefschetz-Sommese theorem [LM, Theorem 1.1] for every zero locus $Z$ of a regular section of $\mathcal{E}$ the restriction homomorphism

$$
\theta_{n-r, 0}: H^{n-r, 0}(X) \rightarrow H^{n-r, 0}(Z)
$$

is injective. This provides the alternative proof of $(*)$ mentioned in the Introduction. As we said there, we are interested in pairs $(X, \mathcal{E})$ as in (1.0) for which (*) is an equality, or equivalently $\theta_{n-r, 0}$ is an isomorphism. The following example will come up again later.

(1.2) Example. Let $\mathcal{F}$ be an ample vector bundle of rank $r+1$ on a smooth projective manifold $Y$ of dimension $n-r \geq 1$. Set $X=\mathbb{P}_{Y}(\mathcal{F})$, let $H$ be the tautological line bundle of $\mathcal{F}$ on $X$ and denote by $\pi: X \rightarrow Y$ the projection. Finally let $\mathcal{G}$ be a spanned vector bundle of rank $r$ on $Y$ and set $\mathcal{E}:=H \otimes \pi^{*} \mathcal{G}$. Then $\mathcal{E}$ is an ample and spanned vector bundle of rank $r$ on $X$. Note that $\mathcal{E}_{F}=\mathcal{O}_{\mathbb{P} r}(1)^{\oplus r}$ for every fibre $F$ of $\pi$. Let $Z$ be the zero locus of a general section of $\mathcal{E}$; then for every fibre $F$, $Z \cap F$ is a linear subspace of dimension $\geq 0$ of $F$. This shows that $\pi_{Z}: Z \rightarrow Y$ is surjective. Moreover, since $Z$ is irreducible, $Z \cap F_{y}$ is a single point for all fibres $F_{y}=\pi^{-1}(y)$ with $y$ outside an algebraic subset of codimension $\geq 2$ of $Y$. Hence $\pi_{Z}: Z \rightarrow Y$ is a birational morphism. Since $H^{0}\left(\Omega_{X}^{n-r}\right) \cong H^{0}\left(\Omega_{Y}^{n-r}\right)$ by construction, this shows that the restriction homomorphism $\theta_{n-r, 0}: H^{0}\left(\Omega_{X}^{n-r}\right) \rightarrow H^{0}\left(\Omega_{Z}^{n-r}\right)$ is in fact an isomorphism. 


\section{Line bundles}

Asking for which pairs $(X, \mathcal{E})$ as in $(1.0)$ equality holds in $(*)$ is a natural, but hard question. This is already clear from the corresponding situation for line bundles. Let $L$ be an ample line bundle on $X$ whose complete linear system $|L|$ contains a smooth element $Z$. For $n=2$ and $L$ spanned, the answer is well known: in fact $\theta_{1,0}$ is an isomorphism if and only if $(X, L)$ is either $\left(\mathbb{P}^{2}, \mathcal{O}_{\mathbb{P}^{2}}(e)\right), e=1,2,\left(\mathbb{P}^{1} \times \mathbb{P}^{1}, \mathcal{O}(1,1)\right)$, or a scroll over a smooth curve. On the contrary, if $L$ is not spanned, some partial results are available ([L, Theorem 1.1], [Fk, Theorem 4.2]), but a complete answer is not yet known.

However in the case of line bundles, the sufficent condition given by Proposition (1.1) becomes also necessary under a moderate further assumption on $L$. In fact we have

(2.1) Proposition. Let $L$ be an ample line bundle on $X$ such that there exists a smooth element $Z \in|L|$ and assume that either $p_{g}(X)=0$ or $h^{0}(L) \geq 2$, Then $\theta_{n-1,0}$ is an isomorphism if and only if $h^{0}\left(K_{X}+L\right)=0$.

Proof. Consider the exact sequence

$$
0 \rightarrow H^{0}\left(K_{X}\right) \stackrel{\iota}{\rightarrow} H^{0}\left(K_{X}+L\right) \rightarrow H^{0}\left(K_{Z}\right) \stackrel{\delta}{\rightarrow} H^{1}\left(K_{X}\right) \rightarrow 0 .
$$

By Serre and Hodge duality $\theta_{n-1,0}$ is an isomorphism if and only if $\delta$ is so and this in turn is equivalent to $\iota$ being an isomorphism, due to the exactness of (2.1.1). Let $p_{g}(X)>0$; then the existence of an obvious finite morphism $\left|K_{X}\right| \times|L| \rightarrow\left|K_{X}+L\right|$ implies the inequality

$$
h^{0}\left(K_{X}+L\right) \geq h^{0}\left(K_{X}\right)+h^{0}(L)-1 .
$$

In view of the assumption this prevents $\iota$ from being an isomorphism. Therefore $p_{g}(X)=0$ and then $\iota$ is an isomorphism if and only if $h^{0}\left(K_{X}+\right.$ $L)=0$.

When $n=3$ and $L$ is ample and spanned, by combining (2.1) with the main result of [S1] and [S2, discussion after Theorem 1.9], we can completely answer our question. In fact we have

(2.2) Theorem. Let $L$ be an ample line bundle on a smooth projective threefold $X$, whose complete linear system $|L|$ contains a smooth surface 
Z. Assume that either $p_{g}(X)=0$ or $h^{0}(L) \geq 2$. Then $\theta_{2,0}$ is an isomorphism if and only if $(X, L)$ is one of the following pairs:

(1) $\left(\mathbb{P}^{3}, \mathcal{O}_{\mathbb{P}}(1)\right),\left(\mathbb{Q}^{3}, \mathcal{O}_{\mathbb{Q}}(1)\right)$, or a scroll over a smooth curve;

(2) a Del Pezzo 3-fold (i. e. , $-K_{X}=2 L$ );

(3) a quadric fibration over a smooth curve;

(4) a scroll over a smooth surface,

or $(X, L)$ admits a reduction $\left(X^{\prime}, L^{\prime}\right)$, which is one of the following pairs:

(5) $\left(\mathbb{P}^{3}, \mathcal{O}_{\mathbb{P}}(3)\right),\left(\mathbb{Q}^{3}, \mathcal{O}_{\mathbb{Q}}(2)\right)$, or

(6) a Veronese bundle, i. e. there exists a morphism $\phi: X^{\prime} \rightarrow B$ onto a smooth curve $B$ such that $2 K_{X^{\prime}}+3 L^{\prime}=\phi^{*} H$ for an ample line bundle $H \in \operatorname{Pic}(B)$.

In particular the assertion is true for $L$ ample and spanned.

For the notion of reduction and all terminology of adjunction theory I refer to $[\mathrm{BS}]$.

\section{Decomposable vector bundles of corank 2}

In this Section vector bundles of corank 2 as in (1.0), satisfying equality in $(*)$, are characterized in the decomposable case. For simplicity we assume that the vector bundles we are dealing with are ample and spanned, though the spannedness requirement could be relaxed to weaker conditions enabling us to apply Theorem (2.2). Let us start by discussing the simplest case of vector bundles of rank 2 on 4 -folds.

So let $\mathcal{E}=\mathcal{L} \oplus \mathcal{M}$ be a decomposable ample and spanned vector bundle of rank 2 on a smooth projective 4-fold $X$. Since both summands $\mathcal{L}, \mathcal{M}$ are ample and spanned we can identify $Z$, the zero locus of a regular section, with the transverse intersection of two general smooth elements $A, B$ in $|\mathcal{L}|$ and $|\mathcal{M}|$ respectively. Set $L:=\mathcal{L}_{B}, M:=\mathcal{M}_{A}$ and note that both $M$ and $L$ are ample and spanned line bundles. By factoring the inclusion of $Z$ in $X$ via $Z \subset A \subset X$, the homomorphism $\theta_{2,0}$ factors through the restriction homomorphisms

$$
H^{2,0}(X) \stackrel{\sigma_{A}}{\longrightarrow} H^{2,0}(A) \stackrel{\theta_{A}}{\longrightarrow} H^{2,0}(Z) .
$$


Note that $\sigma_{A}$ is an isomorphism by the Lefschetz theorem, since $Z \in|M|$ is a smooth ample divisor on $A$. Therefore $\theta_{2,0}$ is an isomorphism if and only if $\theta_{A}$ is so. By factoring the inclusion of $Z \subset X$ via $B$ and arguing in the same way, we conclude that $\theta_{2,0}$ is an isomorphism if and only if both the restriction homomorphisms

$$
H^{2,0}(A) \stackrel{\theta_{A}}{\longrightarrow} H^{2,0}(Z) \quad \text { and } \quad H^{2,0}(B) \stackrel{\theta_{B}}{\longrightarrow} H^{2,0}(Z)
$$

are isomorphisms. Thus Theorem (2.2) applies to both pairs $(A, M)$, $(B, L)$ giving a precise list of possibilities for them. Now, by using known results on ample divisors and the symmetric roles played by $(A, M)$ and $(B, L)$, we can determine all possible triplets $(X, \mathcal{L}, \mathcal{M})$, i. e. , all pairs $(X, \mathcal{E})$ as above, for which $\theta_{2,0}$ is an isomorphism. The argument goes as follows.

First consider $(A, M)$. Since it has to be as in Theorem (2.2) we have to check all the possibilities listed there.

Case (1). Let $(A, M)=\left(\mathbb{P}^{3}, \mathcal{O}_{\mathbb{P}}(1)\right)$. Since $A=\mathbb{P}^{3}$ it must be $(X, \mathcal{L})=\left(\mathbb{P}^{4}, \mathcal{O}_{\mathbb{P}}(1)\right)$ and in this case of course we also get $\mathcal{M}=\mathcal{O}_{\mathbb{P}}(1)$. Let $(A, M)=\left(\mathbb{Q}^{3}, \mathcal{O}_{\mathbb{Q}}(1)\right)$. Since $A=\mathbb{Q}^{3}$ we have two possibilities: $(X, \mathcal{L})$ is either $\left(\mathbb{P}^{4}, \mathcal{O}_{\mathbb{P}}(2)\right)$ or $\left(\mathbb{Q}^{4}, \mathcal{O}_{\mathbb{Q}}(1)\right)$. Looking at $M$ we thus get $\mathcal{M}=\mathcal{O}_{\mathbb{P}}(1)$ in the former case and $\mathcal{O}_{\mathbb{Q}}(1)$ in the latter. Now let $(A, M)$ be a scroll over a smooth curve, say $C$. Since $A$ is a $\mathbb{P}^{2}$-bundle over $C$, a known result of Bădescu on ample divisors [BS, p. 118] says that $(X, \mathcal{L})$ is a scroll over $C$. Since $M$ restricts as $\mathcal{O}_{\mathbb{P}^{2}}(1)$ to the fibres of $A$ we thus see that $\mathcal{M}_{F}=\mathcal{O}_{\mathbb{P}^{3}}(1)$ for every fibre $F$ of $X$.

Case (2). Let $(A, M)$ be a Del Pezzo threefold. Then [LPS, Appendix] applies to determine $(X, \mathcal{L})$ and $\mathcal{M}$. Let $d:=d(A, M)=M^{3}$ be the degree of $(A, M)$. First of all $d \geq 2$, since $M$ is ample and spanned. For $2 \leq d \leq 5$ we get a Del Pezzo 4 -fold $(X, \mathcal{L})$ (i. e. $\left.K_{X}=-3 \mathcal{L}\right)$ of the same type as $A$ with $\mathcal{L}=\mathcal{M}$, plus the 4 triplets $(X, \mathcal{L}, \mathcal{M})=\left(\mathbb{P}^{4}, \mathcal{O}_{\mathbb{P}}(2), \mathcal{O}_{\mathbb{P}}(1)\right),\left(\mathbb{Q}^{4}, \mathcal{O}_{\mathbb{Q}}(1), \mathcal{O}_{\mathbb{Q}}(1)\right),\left(\mathbb{P}^{4}, \mathcal{O}_{\mathbb{P}}(3), \mathcal{O}_{\mathbb{P}}(1)\right)$ and $\left(\mathbb{Q}^{4}, \mathcal{O}_{\mathbb{Q}}(2), \mathcal{O}_{\mathbb{Q}}(1)\right)$; note that the first two of them have already been found in studying case (1). When $d=6$ we get the Del Pezzo 4-fold $X=\mathbb{P}^{2} \times \mathbb{P}^{2}$ with $\mathcal{L}=\mathcal{M}=\mathcal{O}(1,1)$. Case $d=7$ does not lift to dimension 4 . Finally for $d=8$ we get the triplet $(X, \mathcal{L}, \mathcal{M})=$ $\left(\mathbb{P}^{4}, \mathcal{O}_{\mathbb{P}}(1), \mathcal{O}_{\mathbb{P}}(2)\right)$, which, up to exchanging $\mathcal{L}$ with $\mathcal{M}$, was already found in studying case (1).

Case (3). Let $(A, M)$ be a quadric fibration over a smooth curve 
$C$. Then the fibration $f: A \rightarrow C$ extends to a morphism $\tilde{f}: X \rightarrow C$ [BS, Theorem 5.2.3], whose general fibre $F$ contains the general fibre of $f$ as an ample divisor. It thus follows that $\left(F, \mathcal{L}_{F}\right)=\left(\mathbb{P}^{3}, \mathcal{O}_{\mathbb{P}}(2)\right)$ or $\left(\mathbb{Q}^{3}, \mathcal{O}_{\mathbb{Q}}(1)\right)$. In the former case $\tilde{f}: X \rightarrow C$ is a $\mathbb{P}^{3}$ fibration (at this point I cannot yet say that it is a $\mathbb{P}^{3}$-bundle) and $\mathcal{M}_{F}=\mathcal{O}_{\mathbb{P}}(1)$. However, by using the same argument as in [F2, (3.3)] (see also [Z, Case (I) at pp. 194-196]), we see that the existence of such a line bundle $\mathcal{M}$ on $X$ implies that $\left(F, \mathcal{M}_{F}\right)=\left(\mathbb{P}^{3}, \mathcal{O}_{\mathbb{P}}(1)\right)$ for every fibre $F$; in particular it turns out that $X$ is a $\mathbb{P}^{3}$-bundle over $C$. In the latter case $\tilde{f}$ gives to $(X, \mathcal{L})$ the structure of a quadric fibration and we also get $\mathcal{M}_{F}=\mathcal{O}_{\mathbb{Q}}(1)$.

Case (4). Let $(A, M)$ be a scroll over a smooth surface $S$. Since $A$ is a $\mathbb{P}^{2}$-bundle over $S$, several results due to Fania-Sommese and SatoSpindler (see [BS, p. 118]) say that $(X, \mathcal{L})$ is a scroll over $S$ except when $A=\mathbb{P}^{1} \times \mathbb{P}^{2}$, in which case $(X, \mathcal{L})$ is a scroll over $\mathbb{P}^{1}$. In this last case we still have to determine $\mathcal{M}$, which will be done in Lemma (3.1). In the general case, looking at $M$, we immediately see that also $\mathcal{M}$ restricts to the fibres of the scroll projection $X \rightarrow S$ as $\mathcal{O}_{\mathbb{P}^{2}}(1)$.

Finally consider the case when $(A, M)$ admits a reduction $\left(A^{\prime}, M^{\prime}\right)$. In this case there exist a smooth 4-fold $X^{\prime}$ and an ample line bundle $\mathcal{L}^{\prime}$ on $X^{\prime}$ such that $X$ is obtained via a blow-up $\rho: X \rightarrow X^{\prime}$ at a finite set $\left\{x_{1}, \ldots x_{t}\right\}$, and $\mathcal{L}=\rho^{*} \mathcal{L}^{\prime}-\left(E_{1}+\ldots+E_{t}\right)$, where $E_{i}=\rho^{-1}\left(x_{i}\right)$, $i=1, \ldots, t$; moreover $A^{\prime}=\rho(A)$ is a smooth element of $\left|\mathcal{L}^{\prime}\right|[\mathrm{F} 1$, pp. 60-61]. For the pairs in case (5) of Theorem (2.2) we immediately get

i) $\left(X^{\prime}, \mathcal{L}^{\prime}\right)=\left(\mathbb{P}^{4}, \mathcal{O}_{\mathbb{P}}(1)\right)$,

if $A^{\prime}=\mathbb{P}^{3}$, while if $A^{\prime}=\mathbb{Q}^{3}$ then

ii) $\left(X^{\prime}, \mathcal{L}^{\prime}\right)=\left(\mathbb{Q}^{4}, \mathcal{O}_{\mathbb{Q}}(1)\right)$ or

iii) $\left(X^{\prime}, \mathcal{L}^{\prime}\right)=\left(\mathbb{P}^{4}, \mathcal{O}_{\mathbb{P}}(2)\right)$.

On the other hand, in case (6), since $\left(A^{\prime}, M^{\prime}\right)$ is a Veronese bundle over a smooth curve $B$, we have that

iv) $\left(X^{\prime}, \mathcal{L}^{\prime}\right)$ is a scroll over $B$

by the result of Bădescu quoted before. In cases i), ii) and iv) there exists a line $l$ of $\left(X^{\prime}, \mathcal{L}^{\prime}\right)$ passing through $x_{1}$; then, setting $\gamma=\rho^{-1}(l)$ we get

$$
\mathcal{L} \gamma=\rho^{*} \mathcal{L}^{\prime} \gamma-E_{1} \gamma=\mathcal{L}^{\prime} l-E_{1} \gamma=1-1=0,
$$

which contradicts the ampleness of $\mathcal{L}$. So these cases do not occur. In case iii), assuming that $t \geq 2$ and letting $l$ denote the line through $x_{1}$ 
and $x_{2}$ we get in the same way

$$
\mathcal{L} \gamma=\rho^{*} \mathcal{L}^{\prime} \gamma-\left(E_{1}+E_{2}\right) \gamma=\mathcal{L}^{\prime} l-2=0,
$$

contradicting the ampleness of $\mathcal{L}$. Therefore only case iii) with $t=1$ can occur and in this case from $M^{\prime}=\mathcal{O}_{\mathbb{Q}}(2)$ we also get $\mathcal{M}=\mathcal{L}=$ $\rho^{*} \mathcal{O}_{\mathbb{P}}(2)-E_{1}$.

Note that apart from a special situation arising in case (4) the structure of $(A, M)$ has been sufficient to determine $(X, \mathcal{L}, \mathcal{M})$. The special case when $A=\mathbb{P}^{2} \times \mathbb{P}^{1}, M$ giving the structure of a scroll over $\mathbb{P}^{2}$, needs to also consider the pair $(B, L)$. This case is settled by the following

(3.1) Lemma. Let $(A, M)=\left(\mathbb{P}^{2} \times \mathbb{P}^{1}, \mathcal{O}(a, 1)\right)$. Then $(X, \mathcal{L})$ is a scroll over $\mathbb{P}^{1}$ via a morphism $\pi: X \rightarrow \mathbb{P}^{1}$ extending the second projection of $A$ and $\mathcal{M}=a(\mathcal{L}-b F)+F$, where $b$ is a positive integer, $\mathcal{L}_{A}=\mathcal{O}(1, b)$ and $F$ is a fibre of $X$. Moreover $a=1$ or 2 .

Proof. Let $p_{1}$ and $p_{2}$ be the first and the second projection of $A=\mathbb{P}^{2} \times$ $\mathbb{P}^{1}$. We have $M=\mathcal{O}(a, 1)$ for some integer $a \geq 1$. Note that $A=\mathbb{P}(\mathcal{G})$, where $\mathcal{G}=\mathcal{O}_{\mathbb{P}^{2}}(a)^{\oplus 2}$ and $M$ is the tautological line bundle of $\mathcal{G}$. Actually $\mathcal{G}=\mathcal{O}_{\mathbb{P}^{2}}(a) \otimes\left(\mathcal{O}_{\mathbb{P}^{2}}^{\oplus 2}\right)$, hence $M$ is the tautological line bundle of the trivial bundle $\mathcal{O}_{\mathbb{P}^{2}}^{\oplus 2}$ (which is $\mathcal{O}(0,1)$ ) twisted by $p_{1}^{*} \mathcal{O}_{\mathbb{P}^{2}}(a)=\mathcal{O}(a, 0)$. Now the first assertion in the statement follows from the already quoted result of Bădescu [BS, p. 118]. So $X=\mathbb{P}\left(\mathcal{F}^{\prime}\right)$, where $\mathcal{F}^{\prime}$ is an ample vector bundle of rank 4 on $\mathbb{P}^{1}$, which $\mathcal{L}$ is the tautological line bundle of. Let $F$ be a fibre of $\pi$ and recall that $\pi_{\mid A}=p_{2}$. Set $c=\operatorname{deg}\left(c_{1}\left(\mathcal{F}^{\prime}\right)\right)$. Then the Chern-Wu relation gives

$$
\mathcal{L}^{4}=c \mathcal{L}^{3} F=c .
$$

Now look at $\mathcal{M}$. Since $\mathcal{M}_{A}=\mathcal{O}(a, 1), \mathcal{M}$ induces $\mathcal{O}_{\mathbb{P}^{2}}(a)$ on the fibres of $p_{2}$, hence $\mathcal{M}_{F}=\mathcal{O}_{\mathbb{P}^{3}}(a)$. Since $\operatorname{Pic}(X)$ is generated by $\mathcal{L}$ and $F$, we thus get

$$
\mathcal{M}=a \mathcal{L}-\lambda F,
$$

for some integer $\lambda$. In particular, if $a=1$ this shows that $(X, \mathcal{M})$ is a scroll over $\mathbb{P}^{1}$ as well. On the other hand, since $A$ is a product and $\left(A, \mathcal{L}_{A}\right)$ inherits a scroll structure over $\mathbb{P}^{1}$ from that of $(X, \mathcal{L})$, we also have $A=\mathbb{P}(\mathcal{F})$, with $\mathcal{F}=\mathcal{O}_{\mathbb{P}^{1}}(b)^{\oplus 3}$, for some integer $b$ and we can 
assume that $\mathcal{L}_{A}$ is the tautological line bundle of $\mathcal{F}$. So $b>0$. Moreover, from (3.1.1), by using the intersection table of $\mathbb{P}^{2} \times \mathbb{P}^{1}$ we get

$$
c=\mathcal{L}^{4}=\mathcal{L}_{A}^{3}=(\mathcal{O}(1, b))^{3}=3 b .
$$

Similarly, since $M^{3}=(\mathcal{O}(a, 1))^{3}=3 a^{2}$, recalling (3.1.2) we get

$$
3 a^{2}=M^{3}=\mathcal{M}^{3} \mathcal{L}=(a \mathcal{L}-\lambda F)^{3} \mathcal{L}=a^{3} \mathcal{L}^{4}-3 a^{2} \lambda \mathcal{L}^{3} F=a^{3} c-3 a^{2} \lambda .
$$

Since $a>0$, this gives $\lambda=a b-1$, which in view of (3.1.2) proves the assertion on $\mathcal{M}$. Finally note that any smooth element $B \in|\mathcal{M}|$ is a fibration over $\mathbb{P}^{1}$, whose general fibre $\Sigma$ is a smooth surface of $\mathbb{P}^{3}$ of degree $a$; moreover $L=\mathcal{L}_{A}$ induces the hyperplane section bundle on every fibre of $B$. Since also $(B, L)$ has to be one of the pairs listed in Theorem (2.2), this immediately shows that $a \leq 2$. This is immediate in cases (1) - (3). In case (4), $X$, which is a $\mathbb{P}^{3}$-bundle over $\mathbb{P}^{1}$, would be at the same time a $\mathbb{P}^{2}$-bundle over a surface $S$, but this is impossible; otherwise, computing the topological Euler-Poincaré characteristic we should have $8=\chi(X)=3 \chi(S)$, a contradiction. Thus $B \cong \mathbb{P}^{2} \times \mathbb{P}^{1}$, due to a result of Fania-Sommese [BS, p. 118, discussion before Theorem 5.5.3]. This immediately shows that $\Sigma \cong \mathbb{P}^{2}$, hence $a=1$. As to the remaining cases, note that if $(B, L)$ admits a reduction $\left(B^{\prime}, L^{\prime}\right)$, then the fibration of $B$ induces a fibration on $B^{\prime}$, since any exceptional divisor has to be contained in a fibre of $B$. But in case (5) such a fibration cannot exist, while in case $(6)\left(\Sigma, L_{\Sigma}\right)$ should be a Veronese surface, which clearly gives a contradiction. This concludes the proof.

Note that for all pairs $(X, \mathcal{L} \oplus \mathcal{M})$ arising from the above discussion $\theta_{2,0}$ is in fact an isomorphism. Actually when $(X, \mathcal{L})$ is a scroll over a smooth surface $S$ (see case (4)) we noted that even $(X, \mathcal{M})$ is a scroll over $S$, hence this case fits into the more general situation described in Example (1.2). On the other hand, in all the remaining cases we have $h^{2,0}(X)=0$ and looking at the surface $Z$, transverse intersection of two general elements $A \in|\mathcal{L}|$ and $B \in|\mathcal{M}|$, it is immediate to check that $p_{g}(Z)=0$. In conclusion, we proved the following

(3.2) Theorem. Let $\mathcal{E}=\mathcal{L} \oplus \mathcal{M}$ be a decomposable ample and spanned vector bundle of rank 2 on a smooth projective 4 -fold $X$. Then $\theta_{2,0}$ is an isomorphism if and only if $(X, \mathcal{E})$ is one of the following pairs: 
(1) $\left(\mathbb{P}^{4}, \mathcal{O}_{\mathbb{P}}(1) \oplus \mathcal{O}_{\mathbb{P}}(e)\right)$ with $e=1,2$ or 3 ;

(2) $\left(\mathbb{Q}^{4}, \mathcal{O}_{\mathbb{Q}}(1) \oplus \mathcal{O}_{\mathbb{Q}}(e)\right)$ with $e=1$ or 2 ;

(3) $X=\mathbb{P}_{C}(\mathcal{F})$, where $\mathcal{F}$ is an ample vector bundle of rank 4 over a smooth curve $C$ and $\mathcal{L}_{F}=\mathcal{M}_{F}=\mathcal{O}_{\mathbb{P}}(1)$ for every fibre $F$;

(4) $(X, \mathcal{L})$ is a Del Pezzo 4-fold of degree $d, 2 \leq d \leq 6$, and $\mathcal{M}=\mathcal{L}$;

(5) there is a morphism $f: X \rightarrow C$ onto a smooth curve $C$ giving structures of quadric fibrations to both $(X, \mathcal{L})$ and $(X, \mathcal{M})$;

(6) $X=\mathbb{P}_{C}(\mathcal{F})$, where $\mathcal{F}$ is an ample vector bundle of rank 4 over a smooth curve $C$ and $\mathcal{L}_{F}=\mathcal{O}_{\mathbb{P}}(2)$, while $\mathcal{M}_{F}=\mathcal{O}_{\mathbb{P}}(1)$ for every fibre $F$;

(7) $X=\mathbb{P}_{S}(\mathcal{F})$, where $\mathcal{F}$ is a vector bundle of rank 3 over a smooth surface $S$ and $\mathcal{L}_{F}=\mathcal{M}_{F}=\mathcal{O}_{\mathbb{P}}(1)$ for every fibre $F$;

(8) $X$ is $\mathbb{P}^{4}$ blown-up at a single point $x$ and $\mathcal{L}=\mathcal{M}=\rho^{*} \mathcal{O}_{\mathbb{P}}(2)-E$, where $\rho: X \rightarrow \mathbb{P}^{4}$ is the blowing-up and $E=\rho^{-1}(x)$.

By induction it is not hard to extend this result to the case of an ample and spanned vector bundle $\mathcal{E}=\oplus_{i=1}^{n-2} \mathcal{L}_{i}$ on a smooth projective $n$ fold $X$ with $n \geq 5$. In fact passing from dimension 4 to higher dimensions is much easier than passing from dimension 3 to 4 . Actually consider general smooth hypersurfaces $A_{i} \in\left|\mathcal{L}_{i}\right|$, let

$$
\mathcal{E}_{i}:=\mathcal{L}_{1} \oplus \ldots \oplus \widehat{\mathcal{L}}_{i} \oplus \ldots \oplus \mathcal{L}_{n-2},
$$

where $`$ means suppression, and set $E_{i}=\left(\mathcal{E}_{i}\right)_{A_{i}}$. By induction, the pair $\left(A_{i}, E_{i}\right)$ is known and by applying again the already quoted results on ample divisors this allows us to determine $\left(X, \mathcal{L}_{i}\right)$ and $\mathcal{E}_{i}$.

Note that if $n=5$ and $\left(A_{3}, E_{3}\right)$ is as in the last case of Theorem (3.1), by applying again Fujita's result quoted earlier we would get with obvious notation: $\left(X^{\prime}, \mathcal{L}_{3}^{\prime}\right)=\left(\mathbb{P}^{5}, \mathcal{O}_{\mathbb{P}}(1)\right)$ and $\mathcal{L}_{3}=\rho^{*} \mathcal{O}_{\mathbb{P}}(1)-E$, where $\rho: X \rightarrow \mathbb{P}^{5}$ is the blowing-up at $x$ and $E=\rho^{-1}(x)$. Then by taking the inverse image of a line in $\mathbb{P}^{5}$ through $x$ we could immediately contradict the ampleness of $\mathcal{L}_{3}$. This shows that case (8) of Theorem (3.2) does not lift to higher dimensions. Recalling also that there are no Del Pezzo manifolds of degree 6 for $n \geq 5$, the concluding result is the following 
(3.3) Theorem. Let $\mathcal{E}=\oplus_{i=1}^{n-2} \mathcal{L}_{i}$ be a decomposable ample and spanned vector bundle of rank $n-2$ on a smooth projective $n$-fold $X$ with $n \geq$ 5. Then $\theta_{n-2,0}$ is an isomorphism if and only if $(X, \mathcal{E})$ is one of the following pairs:

(1) $\left(\mathbb{P}^{n}, \mathcal{O}_{\mathbb{P}}(1)^{\oplus(n-3)} \oplus \mathcal{O}_{\mathbb{P}}(e)\right)$ with $e=1,2,3$, or $\left(\mathbb{P}^{n}, \mathcal{O}_{\mathbb{P}}(1)^{\oplus(n-4)} \oplus\right.$ $\left.\mathcal{O}_{\mathbb{P}}(2)^{\oplus 2}\right)$;

(2) $\left(\mathbb{Q}^{n}, \mathcal{O}_{\mathbb{Q}}(1)^{\oplus(n-3)} \oplus \mathcal{O}_{\mathbb{Q}}(e)\right)$ with $e=1$ or 2 ;

(3) $X=\mathbb{P}_{C}(\mathcal{F})$, where $\mathcal{F}$ is an ample vector bundle of rank $n$ over a smooth curve $C$ and $\mathcal{E}_{F}=\mathcal{O}_{\mathbb{P}}(1)^{\oplus(n-2)}$ for every fibre $F$;

(4) $(X, \mathcal{H})$ is a Del Pezzo $n$-fold of degree $d, 2 \leq d \leq 5$, and $\mathcal{E}=$ $\mathcal{H}^{\oplus(n-2)}$;

(5) there is a morphism $f: X \rightarrow C$ onto a smooth curve $C$ whose general fibre $F$ is $\mathbb{Q}^{n-1}$ and $\mathcal{E}_{F}=\mathcal{O}_{\mathbb{Q}}(1)^{\oplus(n-2)}$;

(6) $X=\mathbb{P}_{C}(\mathcal{F})$, where $\mathcal{F}$ is an ample vector bundle of rank $n$ over a smooth curve $C$ and, up to reordering the summands of $\mathcal{E}, \mathcal{E}_{F}=$ $\mathcal{O}_{\mathbb{P}}(1)^{\oplus(n-3)} \oplus \mathcal{O}_{\mathbb{P}}(2)$, for every fibre $F$;

(7) $X=\mathbb{P}_{S}(\mathcal{F})$, where $\mathcal{F}$ is a vector bundle of rank $n-1$ over a smooth surface $S$ and $\mathcal{E}_{F}=\mathcal{O}_{\mathbb{P}}(1)^{\oplus(n-2)}$ for every fibre $F$.

In particular, the above results provide the classification of decomposable ample and spanned vector bundles of rank $n-2 \geq 2$ on a projective $n$-fold $X$, with $p_{g}(X, \mathcal{E})=0$. In fact if $(X, \mathcal{E})$ is not as in case (7) of Theorem (3.3) (and (3.2)) we have $p_{g}(X, \mathcal{E})=0$. This follows immediately by identifying $Z$, the zero locus of a regular section of $\mathcal{E}$, with the smooth surface along which $n-2$ general elements $A_{i} \in\left|\mathcal{L}_{i}\right|$ $(i=1, \ldots, n-2)$ intersect transversally. On the other hand, in case (7) we have $p_{g}(X, \mathcal{E})=0$ if and only if $p_{g}(S)=0$, in view of the birational morphism between $Z$ and $S$ induced by the $\mathbb{P}$-bundle projection.

Another obvious consequence of Theorems (3.2) and (3.3) is the following corollary, which generalizes Corollary (3.3) in [S2].

(3.4) Corollary. Let $X$ be a smooth complex projective $n$-fold, $n \geq 3$, and let $\mathcal{L}_{i}$ be an ample and spanned line bundle on $X(i=1, \ldots, n-2)$. Set $\mathcal{E}=\bigoplus_{i=1}^{n-2} \mathcal{L}_{i}$ and let $Z$ be the zero locus of a regular section of $\mathcal{E}$. 
If $p_{g}(X, \mathcal{E})=0$ and $Z$ has nonnegative Kodaira dimension, then $(X, \mathcal{E})$ is as in case (7) of Theorem (3.3), with $p_{g}(S)=0$.

Note that case (7) in Theorem (3.3) (and (3.2)) corresponds to the situation described in Example (1.2) for $n-r=2$.

\section{Final remarks}

It is worth comparing Theorem (3.3) with the classification of ample vector bundles of rank $n-2$ on $X$ whose adjoint bundle is not nef [M1, Theorem, pp. 74-75]. Checking both lists, we see some little discrepancies.

First of all the list in [M1] contains the pair $\left(\mathbb{Q}^{4}, \mathcal{E}:=\mathcal{S} \otimes \mathcal{O}_{\mathbb{Q}}(2)\right)$, where $\mathcal{S}$ is any of the two spinor bundles on $\mathbb{Q}^{4}$; of course such a bundle $\mathcal{E}$ is ample and spanned, but indecomposable. Identify $\mathbb{Q}^{4}$ with the grassmannian $G(1,3)$ of lines of $\mathbb{P}^{3}$, and consider the smooth surface $Z=(s)_{0}$, where $s \in \Gamma(\mathcal{E})$ is general. Then $Z$, representing $c_{2}(\mathcal{E})$, is a congruence of type $(2,3)$, up to exchanging the generators of $H^{4}\left(\mathbb{Q}^{4}, \mathbb{Z}\right)$. Thus $Z$ is a Del Pezzo surface (e. g. see [AS, table at p. 203]), hence $p_{g}(Z)=0=h^{2,0}\left(\mathbb{Q}^{4}\right)$. So the pair $\left(\mathbb{Q}^{4}, \mathcal{E}\right)$ also satisfies equality in $(*)$.

Furthermore, case (8) in [M1, Theorem, p. 75], i. e. the case when $X$ contains a divisor $E$ such that

$$
\left(E, \mathcal{O}_{E}(E), \mathcal{E}_{E}\right)=\left(\mathbb{P}^{n-1}, \mathcal{O}_{\mathbb{P}}(-1), \mathcal{O}_{\mathbb{P}}(1)^{\oplus(n-2)}\right),
$$

does not appear in our lists (except the very special situation described in (8) of Theorem (3.2)). However this discrepancy is reasonable: the fact that $X$ contains a (-1)-hyperplane $E$ as above certainly prevents the adjoint bundle $K_{X}+\operatorname{det} \mathcal{E}$ from being nef but, in principle, it does not exclude the effectiveness.

The following conjecture arises naturally from this comparison.

(4.1) Conjecture. Let $X$ and $\mathcal{E}$ be as in (1.0), with $\mathcal{E}$ ample and spanned of rank $r=n-2$. Assume that $X$ contains no (-1)-hyperplane $E$ such that $\mathcal{E}_{E}=\mathcal{O}_{\mathbb{P}}(1)^{\oplus(n-2)}$. Then $\theta_{n-2,0}$ is an isomorphism if and only if $K_{X}+\operatorname{det} \mathcal{E}$ is not nef.

Note that the corresponding sentence for $r=n-1$ is true, as shown in [LMS, Corollary 2.1]. 
Now come back to the case $\mathcal{E}=\bigoplus_{i=1}^{n-2} \mathcal{L}_{i}$ and, as before, identify $Z$ with the smooth surface along which the general elements $A_{i} \in\left|\mathcal{L}_{i}\right|$ intersect transversally. Corollary (3.4) suggests the following conjecture.

(4.2) Conjecture. Let $X, \mathcal{E}$ and $Z$ be as in (1.0), with $\mathcal{E}$ ample and spanned of rank $r=n-2$. If $p_{g}(X, \mathcal{E})=0$ and $Z$ has nonnegative Kodaira dimension, then $(X, \mathcal{E})$ is as in Example (1.2).

The lists in Theorems (3.2), (3.3) also show that if $p_{g}(X, \mathcal{E})>0$ then equality in $(*)$ occurs only when $(X, \mathcal{E})$ is as in case $(7)$. So, at least for $r=n-2$, this provides an evidence for another conjecture, which I state for any $r \leq n-1$.

(4.3) Conjecture. Let $X, \mathcal{E}$ and $Z$ be as in (1.0), with $\mathcal{E}$ ample and spanned of rank $r \leq n-1$ and suppose that $p_{g}(X, \mathcal{E})>0$. Then $\theta_{n-r, 0}$ is an isomorphism if and only if $(X, \mathcal{E})$ is as in Example (1.2).

Note that this is true for $r=n-1$ [LMS, Theorem]. Conjecture (4.3) could also be formulated in a stronger form, replacing the positivity of $p_{g}(X, \mathcal{E})$ with the requirement that $Z$ has nonnegative Kodaira dimension.

\section{References}

[AS] E. Arrondo and I. Sols, Classification of smooth congruences of low degree, J. reine angew. Math. 393 (198), 199-219.

[BS] M. C. Beltrametti and A. J. Sommese, The Adjunction Theory of Complex Projective Varieties, Exp. Math., vol. 16, de Gruyter, Berlin - New York, 1995.

[F1] T. Fujita, Classification Theories of Polarized Varieties, London Math. Soc. Lecture Note Series, vol. 155, Cambridge Univ. Press, 1990.

[F2] - On adjoint bundles of ample vector bundles, Complex Algebraic Varieties, Proc. Bayreuth, 1990, Lecture Notes in Math., vol. 1507, Springer-Verlag, New York, 1992, pp. 105-112.

[Fk] Y. Fukuma, On polarized surfaces $(X, L)$ with $h^{0}(L)>0, \kappa(X)=2$ and $g(L)=q(X)$, Trans. Amer. Math. Soc. vol. 348 (1996), 4185-4197.

[FkI] Y. Fukuma and H. Ishihara, A generalization of curve genus for ample vector bundles, II, Pacif. J. Math. (to appear). 
[H] R. Hartshorne, Algebraic Geometry, Springer, Berlin - Heidelberg - New York, 1977.

[I] H. Ishihara, A generalization of curve genus for ample vector bundles, I, Comm. Algebra 27(9) (1999), 4327-4335.

[L] A. Lanteri, Algebraic surfaces containing a smooth curve of genus $q(S)$ as an ample divisor, Geom. Dedicata 17 (1984), 189-197.

[LM] A. Lanteri and H. Maeda, Ample vector bundle characterizations of projective bundles and quadric fibrations over curves, Higher Dimensional Complex Varieties (M. Andreatta and Th. Peternell,eds.), Proc. Intern. Conf. Trento, June 1994, De Gruyter, Berlin - New York, 1996, pp. 247-259.

[LMS] A. Lanteri, H. Maeda and A. J. Sommese, Ample and spanned vector bundles of minimal curve genus, Arch. Math. 66 (1996), 141-149.

[LPS] A. Lanteri, M. Palleschi and A. J. Sommese, Del Pezzo surfaces as hyperplane sections, J. Math. Soc. Japan 49 (1997), 501-529.

[M1] H. Maeda, Nefness of adjoint bundles for ample vector bundles, Le Matematiche (Catania) 50 (1995), 73-82.

[M2] Ample vector bundles of small curve genera, Arch. Math. 70 (1998), 239-243.

[SS] B. Shiffman and A. J. Sommese, Vanishing Theorems on Complex Manifolds, Progr. Math., vol. 56, Birkhäuser, Boston - Basel - Stuttgart, 1985.

[S1] A. J. Sommese, On the nonemptiness of the adjoint system on a projective threefold, J. reine angew. Math. 402 (1989), 211-220; Erratum, ibidem 411 (1990), 122-123.

[S2] What we know about the second adjunction mapping, Rend. Sem. Mat. Fis. Milano 68 (1998) (to appear).

[Z] Q. Zhang, A theorem on the adjoint system for vector bundles, Manuscripta Math. 70 (1991), 189-202.

Dipartimento di Matematica "F. Enriques"

Università, Via C. Saldini 50,

I-20133

Milano

Italy

E-mail address: lanteri@vmimat.mat.unimi.it

Recibido: 12 de Febrero de 1999

Revisado: 7 de Junio de 1999 